\title{
A Dynamic Raspberry Pi Sense HAT Multimodality Alerting System by using AWS IoT
}

\author{
T. Manjula*, U. Sreenivasulu and S. Javeed Hussain
}

Department of Electronics and Communication Engineering, MITS, Madanapalle, Chittoor - 517325, Andhra Pradesh, India; manjulathalari@gmail.com, sreenivasuluu@mits.ac.in, consult.drjaveed@gmail.com

\begin{abstract}
Objectives: The main intention of this research is to develop a multimodality system that can monitor the temperature, humidity, barometric pressure, magnetometer, gyroscope, accelerometer values in any applications. Methods: This proposed system uses a wireless Raspberry Pi sense HAT sensor. With this system all the sensor values are obtained and apart from that to design an alerting/notification is being shown by considering temperature parameter. To perform that, the temperature is above predefined limit, which will be notifies by sending out an alert email notification via text messages to user web account by using Amazon Web Services Internet of Thing (AWS IoT) console, and reading the monitor data as visualized in the form of charts using thing speak and also uploads the monitoring data logged into a Google spreadsheet using Wi-Fi adaptor/ LAN to internet access. Simultaneously, all the monitoring data logs are stored in a google spreadsheet to access related google Application Program Interface (APIs) by using OAuth 2.0 protocol. Finding: Obtaining sense Hat sensor data is working on python programming language. Which is a default language is provided by Raspberry Pi. Improvements: This system is used in different applications like cold storage, power plant stations, weather station, homes and chemical laboratories. And also focus on research direction in many fields, such as industry, agriculture by using a wireless sensor node.
\end{abstract}

Keywords: AWS (Amazon Web Services) IoT, OAuth 2.0 Protocol, Python, Raspberry Pi, Raspberry Pi Sense HAT, Thing Speak

\section{Introduction}

Monitoring is being employed in many applications such as like automotive industries, office, homes and soon. Generally, there were lots of major efforts going on in our society. Machines overheating and fire accidents have been forever a problem for various industries ${ }^{1}$. It is observed in most of the industrial applications. Few parameters such as humidity, temperature, pressure and so on, plays a vital role in the smooth functioning of production system. It is quite important to monitor these values for timely action. In this concern, the intention is to design a system that provides the various parameters of the machines using sensors. These sensor values can be monitored from a distant location using IoT (Internet of Thing). It can be connected to billions of devices. It con- sists of a secure communication of user of devices. And also it is provided a lightweight authentication. IoT can be connected to the multiple devices like PCs/laptops, sensors, smart phones to the internet. IoT will upload the monitored data is observed at anytime and anywhere from the internet $\mathrm{t}^{2}=3$.

In early days the work is done on Arduino board. However, it is loaded up to send out of the extremely low price Raspberry pi changed a whole thing. The raspberry pi has gained popularity in many fields, available worldwide, easy to operate, excellent supports and documentation. In educational projects it has proved development, particularly, with applications across over a narrow range of fields. Basically the design of the system consists of easy to works the needs of people the system cost depends on existing hardware and technology $y^{4}$

${ }^{*}$ Author for correspondence 


\subsection{How to Call to Google Spread Sheet and Amazon Web Services Internet of Things with Python}

The entire system uses the python language and it can be easily communicated to Google spread logging web service by OAuth protocol and AWS IoT. Here the allocating data is synced to sensors, devices, PCs by using Internet of thing. AWS IoT affords to attach the hardware device or mobile applications. MQTT (MQ Telemetry Transport) through Internet of thing is used SDK devices enable to connect authenticate and sync the messages. Amazon Simple Notification Sending is a one type of cloud based hardware device notification service, is a flammable, fast and fully managed messaging services and also easily sent out the push notifications, SMS messages and Email ${ }^{5=7}$. This system uses think speak to upload the current machinery monitoring data visualized clearly meaningful graphs by using IoT and API key ${ }^{8}$.

\subsection{Related Work}

In previous days using the various sensors, reading the data of the sensors on the Arduino or raspberry pi board is identified. A basic system to interface raspberry pi to various sensors based on internet of thing. This machine can set a limit range parameter then it will give a control signal in real time. When data are monitored continuously and an alarm message is sent to the owner'. This paper aimsat designing a smart environment monitoring application on raspberry pi by using Internet of thing platform (xively). With this system the DHT22, TMP36 and MQ-7 sensors are interfaced with raspberry pi. It reads the surrounding information through the sensor and uploads to internet access and sent out an alert message of users ${ }^{10}$. This paper aim implemented a smart monitoring system using Internet of Thing. In this system Zigbee IEEE 802.15.4 standard is used to collect the real time sensing data of multi sensors by using IOT then the soil real time data is uploads to send an alert message to user's PC/ laptops ${ }^{11}$.

This system is implemented for Web Based Temperature Monitoring System using ASP server side process $^{122_{13}}$. This system is implemented to provide concepts and a prototype system for industrial automation using Ethernet. With the help of the different sensors which can act as a network node using the LAN to SPI communication ${ }^{14}$. This system has proposed to designed temperature monitoring system in cold chain based on RFID $^{15}$. The proposed intended for home automation by using Raspberry pi and raspberry pi has interfaced with Pi camera for capturing images, and then send the image to user id with internet access ${ }^{16}$. This system had intended for attendance management and user security purposes using raspberry pi 2 board and camera. Here raspberry pi camera interfaced with pi board. For face recognition and detection working on Eign face algorithm method using Ethernet ${ }^{17}$.

\section{System Design}

The proposed system is comprised of both software and hardware design. When, we consider the hardware design. It is comprised of Raspberry Pi, Sense_hat sensor. The detailed explanation of hardware is specified.

\subsection{Hardware System Design}

\subsubsection{Raspberry Pi 2 board}

The Raspberry pi board was developed by the raspberry pi foundation in the UK for the use of computer science education. And the single board computer is at the heart of the system, small, low cost, powerful and lightweight credit-card sized single-board computer. It is an ARM based computer which performs many operations does that a desktop PC. The second generation of raspberry pi is used in this project is indicated in Figure 1. It is six times faster than in the first generation of raspberry pi board.

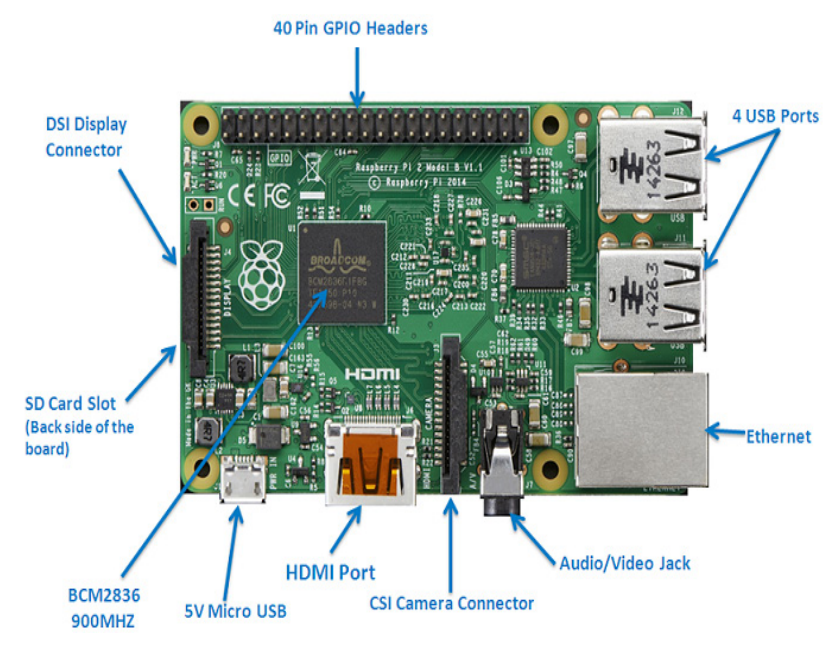

Figure 1. Raspberry Pi 2 board. 
The model B computer is the most popular update version of Pi. The Raspberry pi board supports Linux, ARM, Qtonpi, Mac operating systems. It consists of an ARM cortex-A7 processor, which runs at $900 \mathrm{MHZ}$ clock speed, now available four USB ports of pi, 1 GB of RAM and same a video core IV GPU. It has one HDMI output, one video and audio output, one $100 \mathrm{Mbps}$ Ethernet port and also 40 GPIO (General purpose input/output) pins and it needs the power cable, an external micro SD (Secure Digital) card used for operating system/booting purpose.

\subsubsection{Raspberry Pi Sense_hat board}

The Raspberry pi sense_hat is an advanced a wireless sensor board, interfacing with raspberry pi board and integrated with different sensors like Temperature, Humidity, Barometric Pressure, Gyroscope, Accelerometer, and Magnetometer. In the last few years it was used in Astro pi space for monitoring the weather condition in the workshop areas as indicated in the Figure 2.

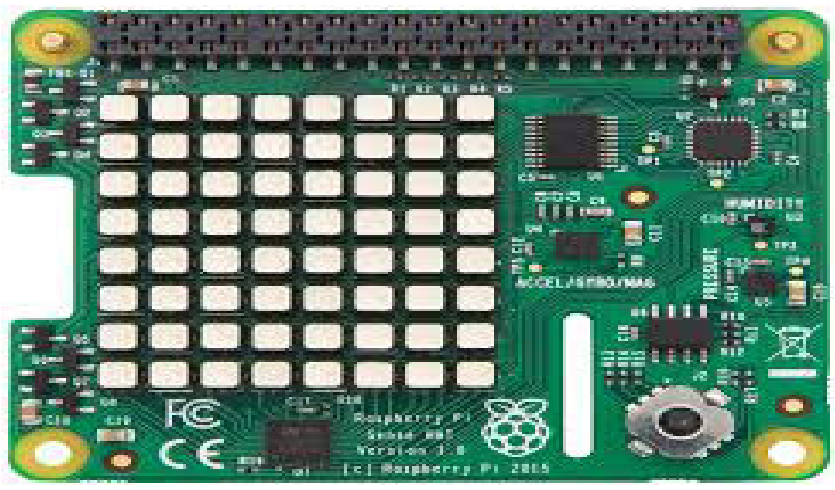

Figure 2. Raspberry Pi Sense HAT board.

It supports to only raspberry pi boards, its $40 \mathrm{GPIO}$ pins are interfaced on the top of the raspberry pi board. This will be done the work on various applications. It has a low cost, small size and light weight. It can be used for various experiments and even games. And it has additional features as a five button joystick and an 8X8 RGB LED (Light Emitting Diode) display because it emits light and diode means current can flow through only one direction. And sense hat board LED arranged in a matrix, LED has a red, green and blue component. We can create any colour or shade by using 0 to 255 matrixes.

Technical Specifications of Raspberry Pi Sense HAT

- Gyroscope - angular rate sensor: $\pm 245 / 500 / 2000 \mathrm{dps}$
- Accelerometer - Linear acceleration sensor: $\pm 2 / 4 / 8 / 16 \mathrm{~g}$

- Magnetometer - Magnetic Sensor: $\pm 4 / 8 / 12 / 16$ gauss

- Barometer: 260 - $1260 \mathrm{hPa}$ absolute range (accuracy depends on the temperature and pressure, $\pm 0.1 \mathrm{hPa}$ under normal conditions)

- Temperature sensor (Temperature accurate to \pm $2{ }^{\circ} \mathrm{C}$ in the $0-65^{\circ} \mathrm{C}$ range)

- Relative Humidity sensor (accurate to $\pm 4.5 \%$ in the $20-80 \% \mathrm{rH}$ range, accurate to $\pm 0.5^{\circ} \mathrm{C}$ in $15-40{ }^{\circ} \mathrm{C}$ range)

Overview of Raspberry pi sense hat board is attached to the top of the raspberry pi 2 board as shown in Figure 3.

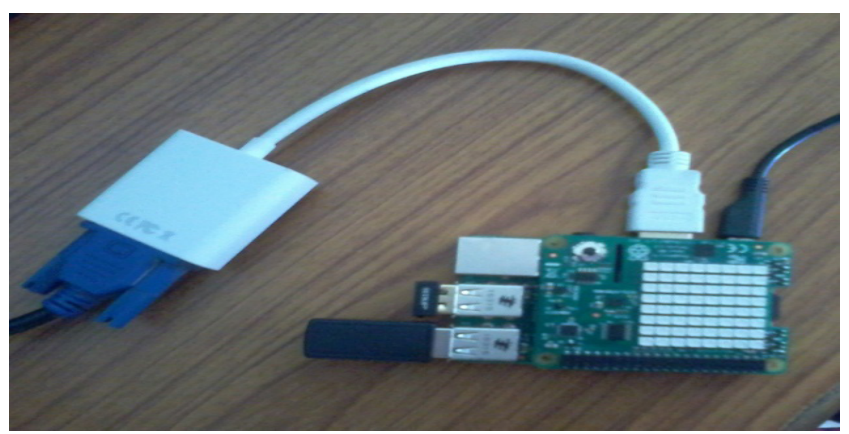

Figure 3. Overview of Raspberry Pi sense HAT.

\subsubsection{Power Supply}

The proposed system is requiring the power supply range from $800 \mathrm{~mA}$. This system uses a USB connection to power itself.

\subsubsection{Implementation of Proposed System Hardware Setup}

In this section the hardware setup of the proposed system raspberry pi board is requiring the input components are HDMI (High Definition Multimedia Interface) to VGA (Video Graphics Array) Wireless Keyboard \& Mouse, WI-FI adaptor, SD card is given the connection as shown in Figure 4. Next to provide power supply of the proposed system is shown in Figure 5.

\subsubsection{Raspbian OS}

In this hardware model Raspbian Jessie version is used. The proposed method sense_hat is supported on python programming language, which includes different python 
packages. It runs LXDE (Lightweight X11 Desktop Environment) as the desktop environment.
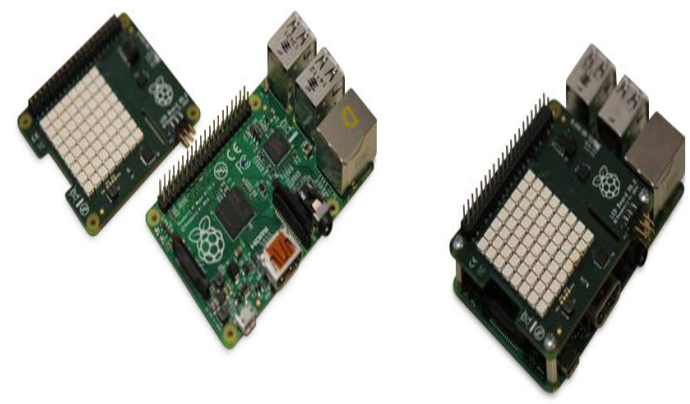

Figure 4. Input connections of Pi board.

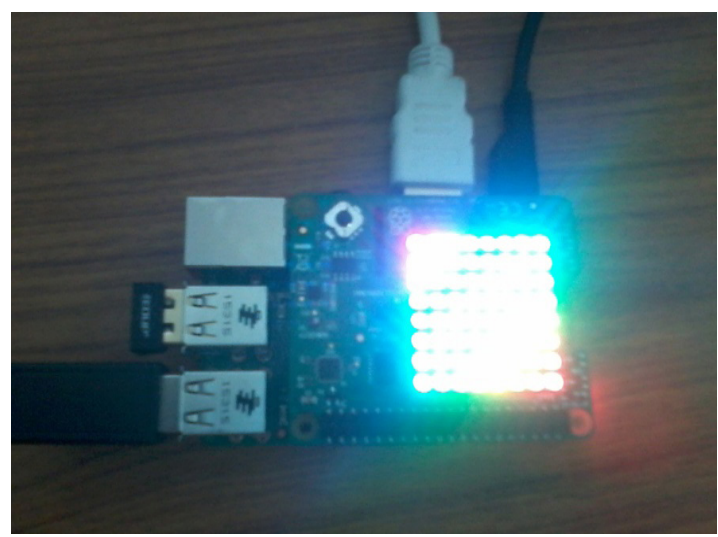

Figure 5. Provide power supply of proposed system.

\section{Steps to install Raspbian Jessie OS:}

1. First step to install the 'WIN32DiskImager' application on our windows system.

2. The next step to download raspberry pi with sense hat board supported by Raspbian Jessie OS.

3. Insert the SD card adaptor into raspberry pi board.

4. By using the WIN32DiskImager application to burn us into an SD card as shown in Figure 7.

\subsection{Software System Design}

\subsubsection{Installation}

In this section the whole hardware system working depends on the software system, the proposed system software design is required to update kernel services as shown in Figure 8. Here some library files are required to initialize for update data can be extracted from the hardware. The micro card computer board is arranged with UNIX kernel services, which can be added to supports the sense_hat characteristics. To update the kernel services of raspberry pi is specified as shown in the below syntax.

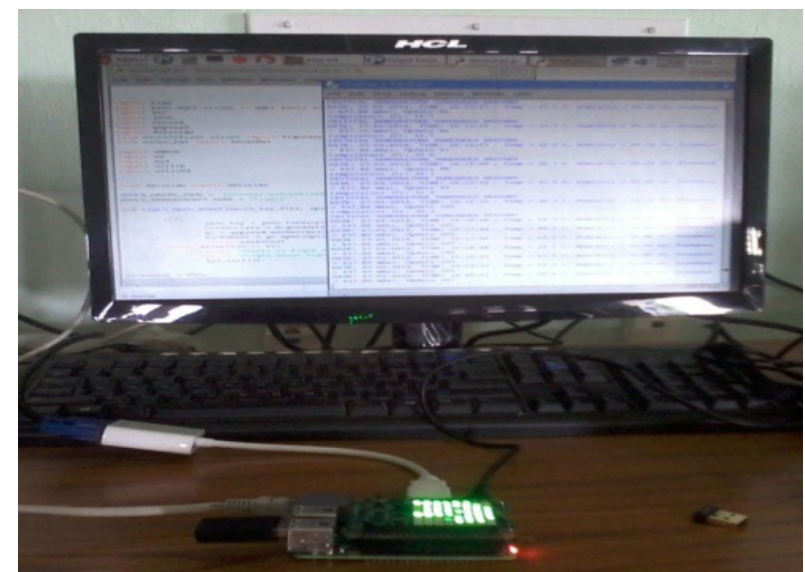

Figure 6. Hardware setup photography.

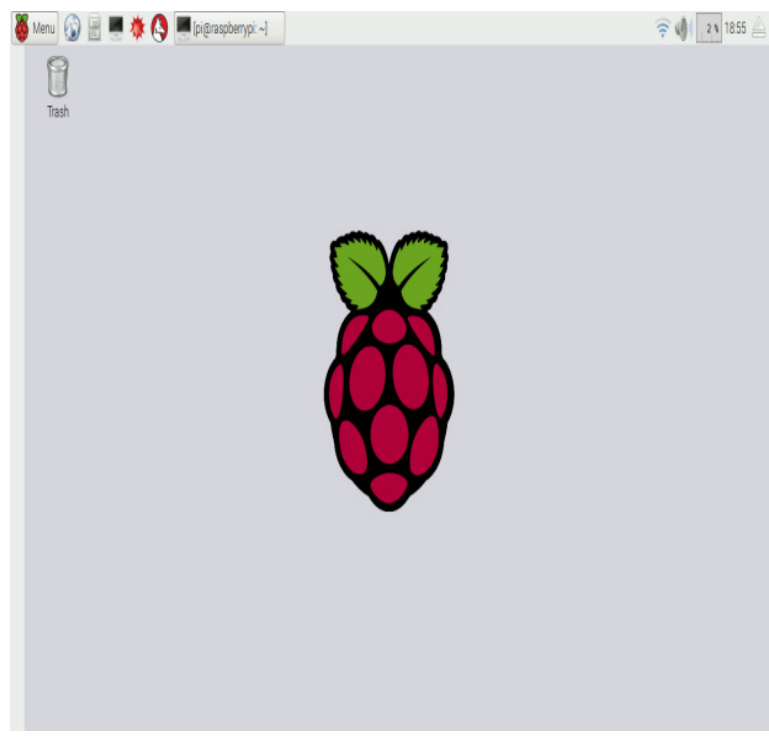

Figure 7. Installed Raspbian Jessie OS.

\section{"Sudo apt-get update"}

After installation of kernel services updating, next to install the sense_hat sensor related certain packages are up-to-date in the kernel. From the below syntax is used to update sense_hat packages in the LX terminal.

\section{"Sudo apt-get install sense hat"}

Finally, the raspberry pi is to reboot and performed to certify that the updating process can obtain necessary changes in the kernel according to the installed packages. Here the rebooting operation is performed used to the following command as shown in below 


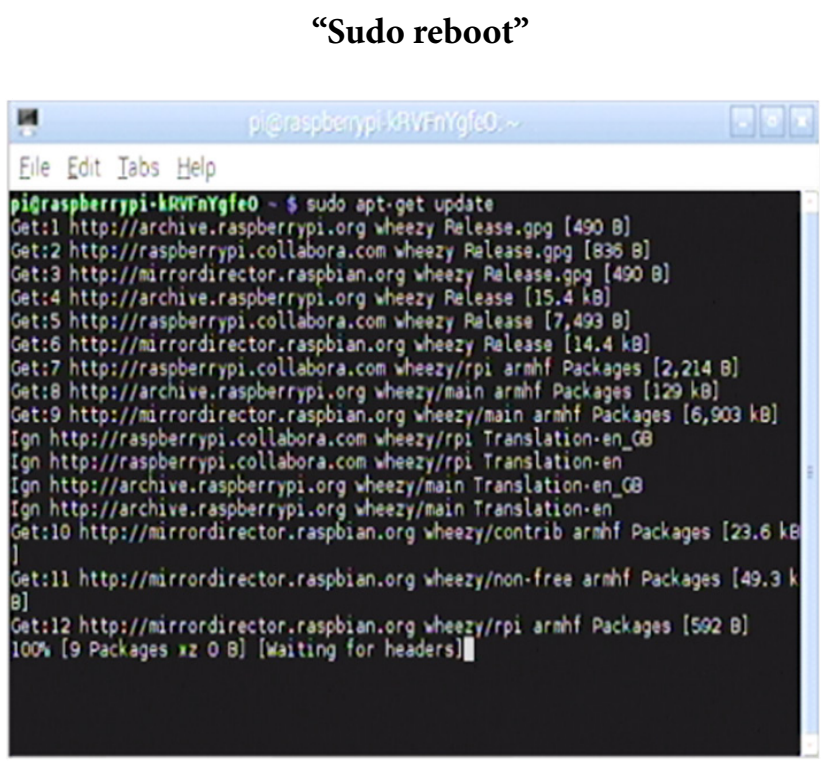

Figure 8. To update the kernel services of Raspberry pi installed.

\section{AWS IOT}

AWS IoT is IoT platform that's designed to enable meaningful connections between people and things. The features of IoT is real-time data collection, data analysis, data processing of the position information, data visualization, message transmission, etc. using a connected SNS, via an open source API to support a various platforms Amazon Web Services of Internet of thing is permits secured. MQTT works on the AWS cloud. It provides a bi-directional communication between the IoT devices like embedded devices or smart appliances, sensors and actuators. It can be easy to communicate between the things and peoples. The main advantage is this application is to upload the real time data, easy to analysis and to locate the processing information. It is helpful for various applications, easy to transfer the data from raspberry pi and Arduino (embedded devices).

\section{Thing Speak}

The Thing speak is an IoT platform providing for different services. Its implementation is easy to communicate between the things and peoples. The thing speak is one type of core element, supports various languages (such as C, Node.js, and python). The sense HAT sensor is sensing the multi parameters real time data is visualized in the form of the charts in thing speak. This platform is tar- geted for IoT applications and the main feature is a real time data is easily to collect, analysis, locate the processing, and also data visualization. It has uses in different environments.

\section{Google Spreadsheet}

Google drive APIs uses the OAuth 2.0 protocol for authentication and to create a service account under a Google mail, it will be given access the spreadsheet. Credentials are required the Server account and API key to access the spreadsheet. It requires oauth 2 client libraries are installed for python.

\section{Proposed System}

The proposed system is possible can sense the humidity, temperature, barometric pressure, accelerometer, gyroscope and magnetometer related values in any application using raspberry pi with internet connection and throw that data in web services. The block diagram of the proposed systemis shown in Figure 9.

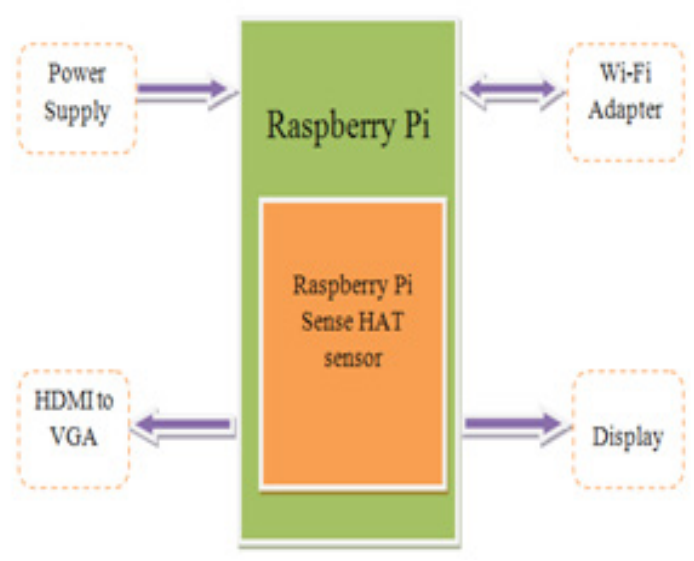

Figure 9. Block diagram of proposed system.

In this proposed system block diagram is consists of the raspberry pi sense HAT sensor is attached to the top of the small size computer board. The raspberry pi is accessing a wireless sense HAT sensor values and processing them to transfer data by using IOT. If the temperature value is to cross the above predefined limit $\left(35^{\circ} \mathrm{C}\right)$ to communicate the raspberry pi and sent an email notification to the user web account and also the sensing information are stored in the raspberry pi board. At a time, the sensor data can be viewed in thing speak and logging all 
the measurement data into a Google spreadsheet using a $\mathrm{Wi}-\mathrm{Fi}$ adaptor. Here the whole system works done in python environment. Simultaneously, all the measurement data are logged into a Google spreadsheet.

\section{Algorithm}

First of all, we have to register an account of the Raspberry Pi device on AWS IoT. Next download certificate according that to allow communication through the IoT cloud. By using python these certificates must be placed in the parent directory of the program. When we connect the IoT console it contains like secured credentials. In cloud the Raspberry Pi should have resources to be created, next device thing is to be created and given the same name of Raspberry Pi. To create this thing is not required of extra attributes. Certificates can be generated by using python programming, and alternatively selecting the SDK like Node Js, and to be created unique certificates for pi device is indicated in Figure 10.

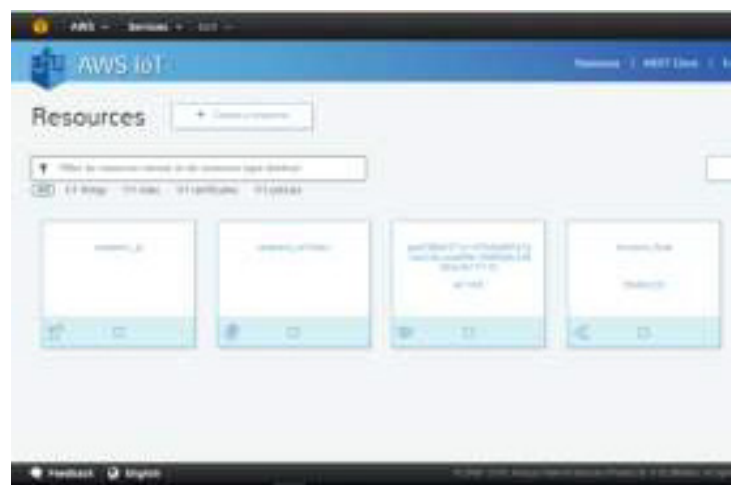

Figure 10. AWS IoT console.

By using Winscp program to transfer needed libraries and certificates to the pi memory. Its communication is done by using the IP address of the raspberry pi. It provides the bidirectional transfer of files. It is acquired by python, programming and results a private / public certification authority key file. To create in addition IoT certificates AWS requires a license from Symantec.

Next, we have to register raspberry pi device on thing speak and get an API key to enable communication through the cloud. In the main module of the program to enter the API key, which is used to communicate with Thing speak. Here, the small computer pi board has to create the resource account in the cloud. To create a New Channel thing by any preferred name. Data can be imported from a channel feed in CSV format. The flow chart of the proposed system is shown in Figure 11.

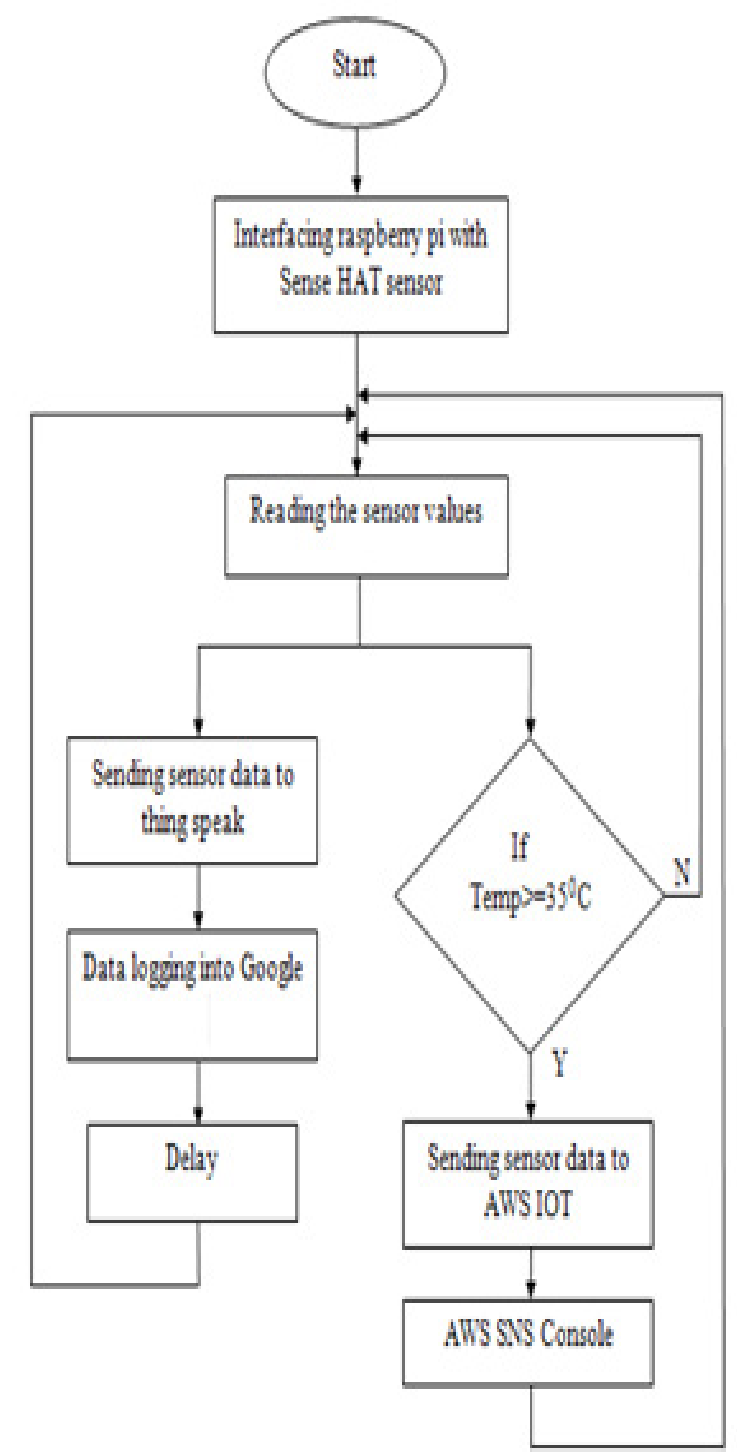

Figure 11. Flow chart of proposed system.

In a closed chamber to attach a sensor in the near of machinery and continuously reading the monitoring machinery temperature, humidity and barometric pressure values. If the sensor detected temperature value above 35 degrees Celsius and will notifies the information, it will allocate a string "CRITICAL TEMPERATURE THERESHOLD REACHED" to the SNS console and sent an email immediately to user id, and also uploads the temperature, and humidity and barometric pressure data visualize the real time monitoring data through graphically charts. And also uploads the temperature, humidity and Barometric Pressure values into a spreadsheet of user.

It can log temperature, humidity and barometric data in the spreadsheet and having the security credentials. 


\section{Experimental Results}

In the proposed system, the multimodality values of the system sensors like temperature, pressure, humidity are obtained and shown in Figures 12-13. The proposed method is defining the experimental results will be seen after running the code. If the temperature value detected above $35^{\circ} \mathrm{C}$ range in the closed chamber at industries which will notifies an alert text message email notification to the user. In a web browser, email is opened and we can get an email notification as shown in the Figure 14.

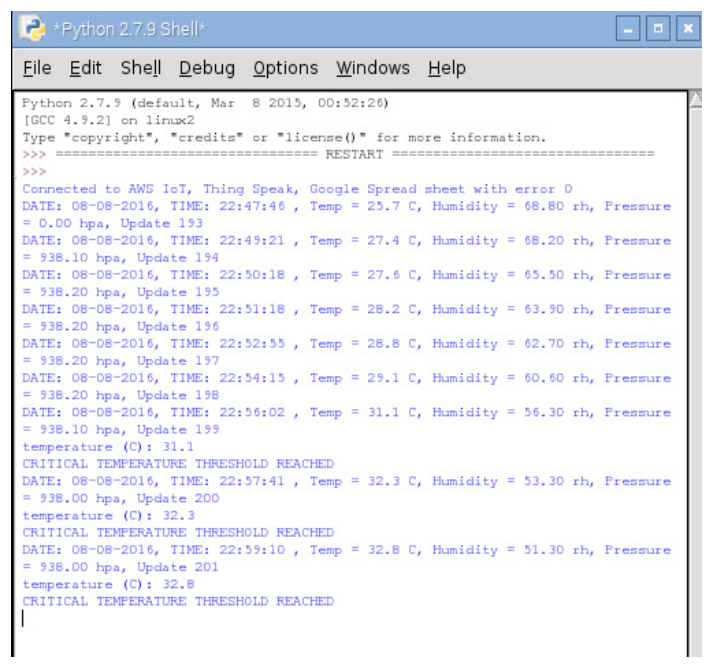

Figure 12. Output results shown in python shell.

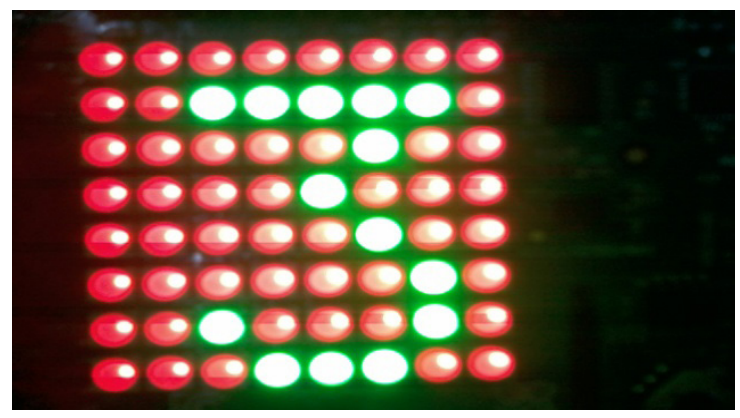

Figure 13. To display the output on LED matrix.

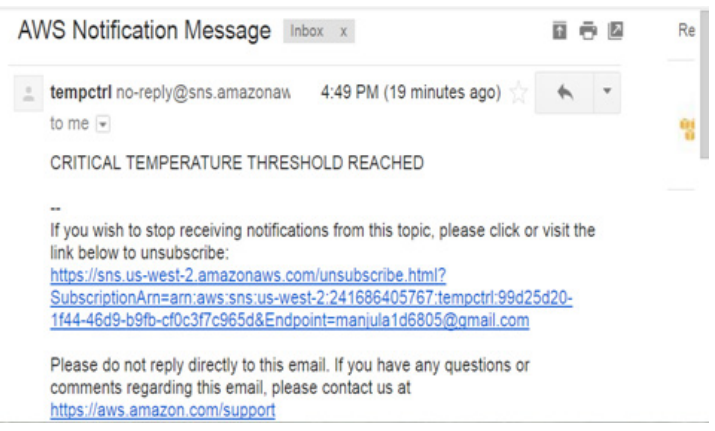

Figure 14. Email in the web browser.
In our proposed method, we can monitor the machinery parameters in the closed chamber by using IOT. The machinery parameters values stored in web server on the cloud. These parameters can be visualized by using separate address and output results are shown graphs is indicated in Figure 15 from a web server on the cloud.

The sensor monitoring data is logged into a google spreadsheet to connect the IoT is indicated in Figure 16. It is easy to analyze the variation of data periodically. By this type of proposed system can be utilized for different applications such as cold storage, and food industry areas etc.
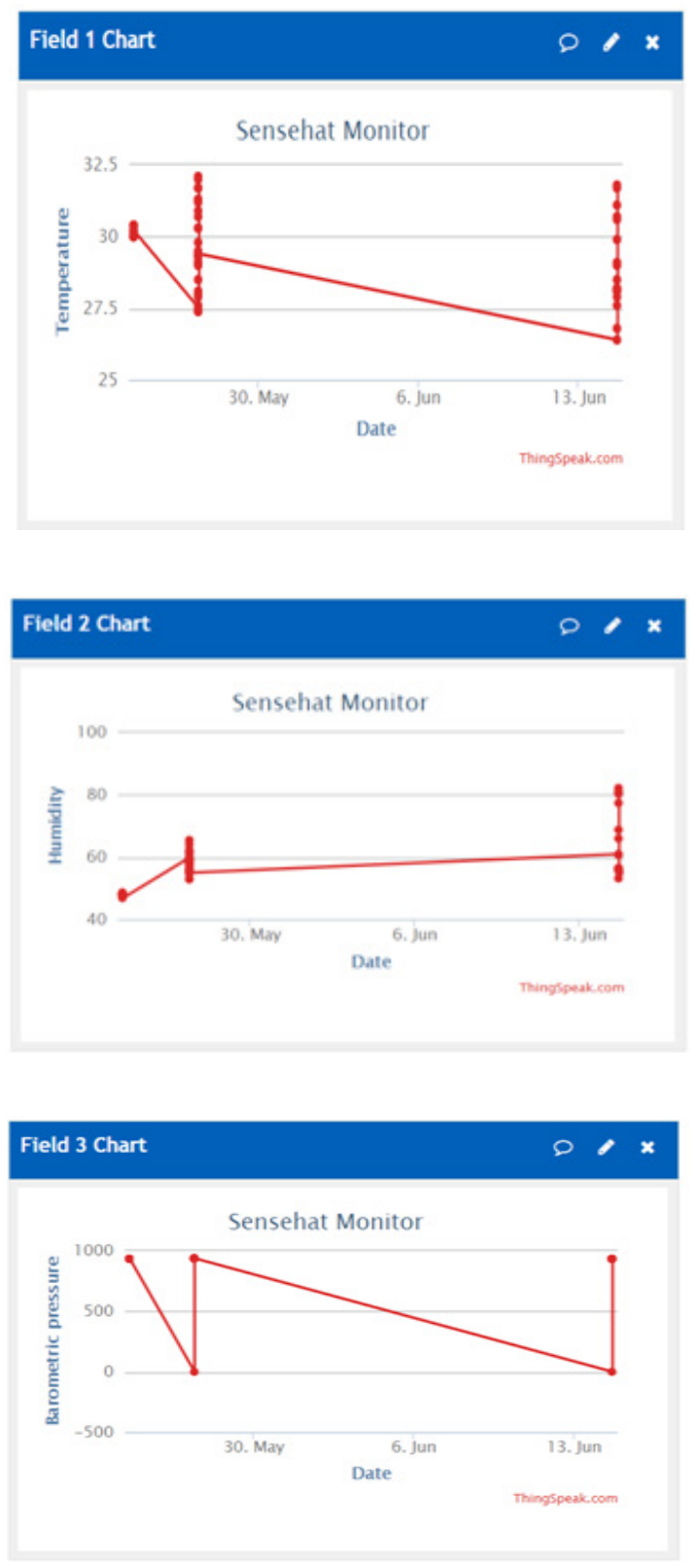

Figure 15. Output results from Web Server. 


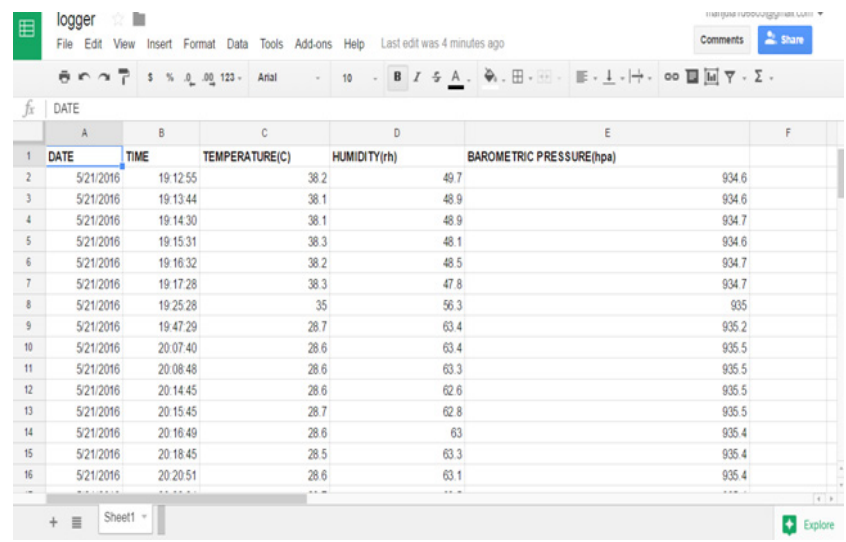

Figure 16. Google spreadsheet data logging.

\section{Conclusion and Future Scope}

The proposed system can be utilized as multimodality system mainly focuses on different application. We can design and development of real time monitoring of machinery parameters in a closed chamber and the temperature value is detected above predefined limit will alert an email notification in IOT environment. This system uses raspberry pi 2 model B and Internet of Thing technology and sense HAT sensor. These devices are cheaper and simpler, capable of processing, more efficient, analyzing, and sending and also viewing of data on the web server.

This can implement is employed in banks, homes, offices, power plant stations, weather monitoring stations, chemical laboratories, and medical areas, etc. And the data are viewed anywhere in the world. An alarm system is future improvement of proposed system which notifies by a loud alarm.

\section{References}

1. Sandeep V, Gopal K L, Naveen S, Amudhan A, Kumar LS. Globally accessible machine automation using raspberry pi based on Internet of Things. International Conference on Advances in Computing, Communications and Informatics, India; 2015.

2. Feng X, Laurence TY. Internet of Things. Microsoft. 2012; 25:1101-2.

3. Raza S. Lithe: Lightweight secure CoAP for the internet of things. IEEE Sensors Journal. 2013; 13(10):3711-20.
4. About Raspberry Pi 2 [Internet]. 2015 Feb. Available from: www.raspberrypi.org.

5. AWSIOT [Internet]. 2016. Available from: docs.aws.amazon.com/iot/latest/developerguide/what-is-aws-iot.html.

6. Yao X, Han X. A light weight multicast authentication mechanism for small scale IoT applications. 2013; 13(10):3693-701.

7. Sruthi MM, Mary LJ. Smart pollution detection and tracking system embedded with AWS IOT cloud. International Journal of Advanced Research in Computer Science and Software Engineering. 2016; 6(4):1-20.

8. ThingSpeak [Internet]. 2016. Available from: http://thingspeak.com/docs/tutorial.

9. Karankumar MD, Shreya MB, Kapil RS. Analysis of TOI (Things of Internet) industrial monitoring system on Raspberry Pi platform. International Journal of Computer Science and Mobile Applications. 2014; 2(11):33-40.

10. Ibrahim M, Elgamri A, Babiker S, Mohamed A. Internet of things based smart environmental monitoring using the raspberry-pi computer. 2015 Fifth International Conference on Digital Information Processing and Communications (ICDIPC), 2015 Oct 7-9; 2015 Nov 12.

11. Tintoiya AA, Shah MSS. IOT based smart monitoring system. Journal of Emerging Technologies and Innovative Research. 2015; 2(3):1568-77.

12. Kasin M, Ismil MN, Yahaya CKHCK. Web based temperature monitoring system. International journal of Multidisciplinary Sciences and Engineering. 2011; 1(2):729-36.

13. Breniuc L, Haba CG. Web-based monitoring and control of a temperature measurement system. Proceedings of the $2^{\text {nd }}$ on-line Workshop on Tools for Education in Measurement, Tampere, Finland; 2002. p. 11-15.

14. Gore RP, Mobeen SA. Industrial process parameter control using ethernet. International Research Journal of Engineering and Technology. 2015 Sep; 2(6):676-81.

15. Yan B, Lee D. Application of RFID in cold chain temperature monitoring system. ISECS International Colloquium on Computing, Communication, Control, and Management. 2009; 2.

16. Jain S, Vaibhav, Goyal L. Raspberry Pi based interactive home automation system through E-mail. 2014 International Conference on Optimization, Reliabilty, and Information Technology (ICROIT); 2014 Feb. p. 277-80.

17. Gaddam SS, Ramesh NVK. Attendance management and user security system's based on Eign faces algorithm using 